\title{
MACREL: antimicrobial peptide screening in genomes and metagenomes
}

\author{
Célio Dias Santos-Junior (i) 1,2, Shaojun Pan (가 1,2, Xing-Ming Zhao (i) 1,2, and \\ Luis Pedro Coelho iㅣ, 1,2,
}

${ }^{1}$ Institute of Science and Technology for Brain-Inspired Intelligence, Fudan University, Shanghai, 200433, China

${ }^{2}$ Key Laboratory of Computational Neuroscience and Brain-Inspired Intelligence, Ministry of Education, China

*to whom correspondence should be addressed: luispedro@big-data-biology.org

\section{ABSTRACT}

Motivation: Antimicrobial peptides (AMPs) have the potential to tackle multidrug-resistant pathogens in both clinical and non-clinical contexts. The recent growth in the availability of genomes and metagenomes provides an opportunity for in silico prediction of novel AMP molecules. However, due to the small size of these peptides, standard gene prospection methods cannot be applied in this domain and alternative approaches are necessary. In particular, standard gene prediction methods have low precision for short peptides, and functional classification by homology results in low recall.

Results: Here, we present Macrel (for metagenomic AMP classification and retrieval), which is an end-to-end pipeline for the prospection of high-quality AMP candidates from (meta)genomes. For this, we introduce a novel set of 22 peptide features. These were used to build classifiers which perform similarly to the state-of-the-art in the prediction of both antimicrobial and hemolytic activity of peptides, but with enhanced precision (using standard benchmarks as well as a stricter testing regime). We demonstrate that Macrel recovers high-quality AMP candidates using realistic simulations and real data.

Availability: Macrel is implemented in Python 3. It is available as open source at https://github.com/BigDataBiology/macrel and through bioconda. Classification of peptides or prediction of AMPs in contigs can also be performed on the webserver: http://big-data-biology.org/software/macrel.

\section{Introduction}

Antimicrobial peptides (AMPs) are short proteins (containing fewer than 100 amino acids) that can decrease or inhibit bacterial growth. Given the dearth of novel antibiotics in recent decades and the rise of antimicrobial resistance, prospecting naturallyoccurring AMPs is a potentially valuable source of new antimicrobial molecules (Theuretzbacher et al., 2019). The increasing number of publicly available metagenomes and metatranscriptomes revealed a multitude of microorganisms so far unknown, harboring an immense biotechnological potential (Pascoal et al., 2020; Bernard et al., 2018). It presents an opportunity to use these (meta)genomic data for finding novel AMP sequences. However, methods that have been successful in prospecting other microbial functionality, cannot be directly applied to small genes (Saghatelian and Couso, 2015), such as AMPs. In particular, there are two major computational challenges: the prediction of small genes in DNA sequences (either genomic or metagenomic contigs) and the prediction of AMP activity for small genes using homology-based methods.

Current automated gene prediction methods typically exclude small open reading frames (smORFs) (Miravet-Verde et al., 2019), as the naïve use of the methods that work for larger sequences leads to unacceptably high rates of false positives when extended to short sequences (Hyatt et al., 2010). A few recent large-scale smORFs surveys have, nonetheless, shown that these methods can be employed if the results are subsequently analyzed to eliminate spurious gene predictions. This procedures reveal biologically active prokaryotic smORFs across a range of functions (Miravet-Verde et al., 2019; Sberro et al., 2019).

Similarly, the prediction of AMP activity requires different techniques than the homology-based methods that are applicable for longer proteins (Huerta-Cepas et al., 2017). In this context, several machine learning-based methods have demonstrated high accuracy in predicting antimicrobial activity in peptides, when tested on curated benchmarks (Xiao et al., 2013; Meher et al., 2017; Lata et al., 2010; Thakur et al., 2012; Sharma et al., 2016; Bhadra et al., 2018). However, to be applicable to the task of extracting AMPs from genomic data, an AMP classifier needs to be robust to gene mispredictions and needs to be benchmarked in that context. In particular, realistic evaluations need to reflect the fact that most predicted genes are unlikely to 
have antimicrobial properties.

\section{Results}

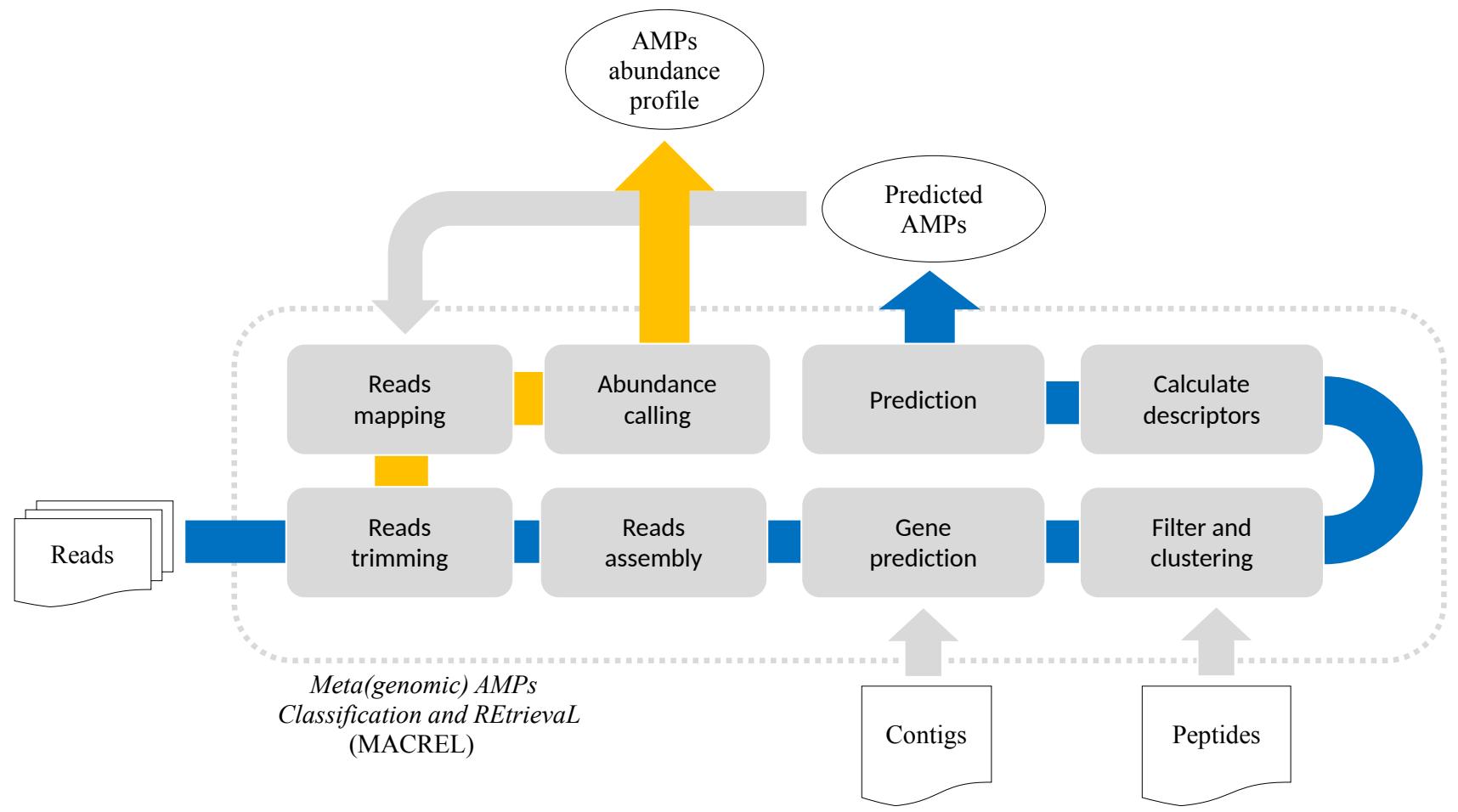

Figure 1. (Meta)genomic AMPs Classification and REtrievaL: Macrel pipeline. The blue arrows show the Macrel workflow from the processing of reads until AMP prediction. The user can alternatively provide contigs or peptide sequences directly, skipping the initial steps of the short-read pipeline. The yellow arrow shows the abundance profiling of AMPs using Macrel output and reads.

\subsection{Macrel: (Meta)genomic AMPs Classification and REtrievaL}

Here, we present Macrel (for (Meta)genomic AMPs Classification and REtrievaL, see Fig. 1), a simple, yet accurate, pipeline that processes either genomes or metagenomes/metatranscriptomes and predicts AMP sequences. We test Macrel with standard benchmarks for AMP prediction as well as both simulated and real sequencing data to show that, even in the presence of large numbers of (potentially artifactual) input smORFs, Macrel still outputs only a small number of high-quality candidates.

Macrel can process metagenomes (in the form of short reads), (meta)genomic contigs, or peptides. If short reads are given as input, Macrel will preprocess and assemble them into larger contigs. Automated gene prediction then extracts smORFs from these contigs which are classified into AMPs or rejected from further processing (see Fig. 1 and Methods). Putative AMPs are further classified into hemolytic or non-hemolytic. Unlike other pipelines (Jhong et al., 2019), Macrel can not only quantify known sequences, but also discover novel AMPs.

Macrel is also available as a webserver at http://big-data-biology.org/software/macrel, which accepts both peptides and contig sequences, and retrieves AMPs coded by their own genes.

\subsection{Novel set of protein descriptors for AMP identification}

Two binary classifiers are used in Macrel: one predicts AMP activity and another the hemolytic activity (which is invoked only for putative AMPs). These are feature-based classifiers and use a set of 22 variables that capture the amphipathic nature of AMPs and their propensity to form transmembrane helices (see Suppl. Table S1). 
Peptide sequences can be characterized using local or global features: local features depend on the order of the amino acids, while global ones do not. Local features have been shown to be more informative when predicting AMP activity and its targets, while global features are more informative when predicting the potency of a given AMP (Bhadra et al., 2018; Fjell et al., 2009; Boone et al., 2018). Thus, Macrel combines both, including 6 local and 16 global features (see Methods and Suppl. Table S1):

- Free energy transition (FET) (3 local features). This is a novel feature group, which was designed to capture the fact that AMPs usually fold from random coils in the polar phase to well-organized structures in lipid membranes (Nagarajan et al., 2019). Each amino acid is assigned to one of three groups of increasing free-energy change (von Heijne and Blomberg, 1979). The three features consist of the position of the first amino acid in each group, normalized to the length of the sequence (see Fig. 2). Earlier works had shown that the N-terminal is particularly informative for determining AMP activity (Bahar and Ren, 2013; Bhadra et al., 2018). We adopted the fractional position encoding from the more general CTD framework (Dubchak et al., 1995, 1999).

a)

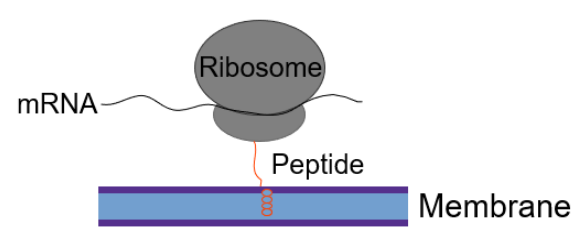

b)

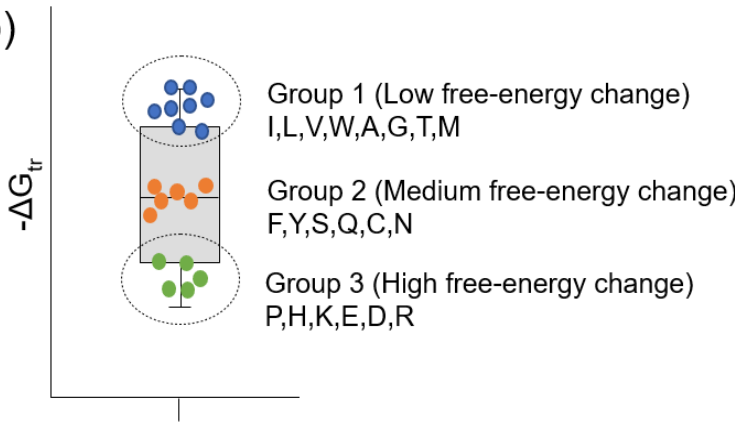

c)

$$
D_{(0)}^{\text {Group N }}=\frac{\text { First residue of group } N}{\text { Length }}
$$

Microbisporicin A1 (24 res.)

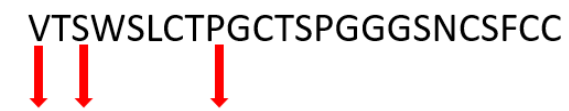

Pos. $\Rightarrow 123456789$...

$$
D_{(0)}^{G 1}=\frac{1}{24} \quad D_{(0)}^{G 2}=\frac{3}{24} \quad D_{(0)}^{G 3}=\frac{9}{24}
$$

Figure 2. The FET-Free energy transition-measures estimates the propensity of peptides to fold when transferring from water to the membrane. The estimated change in the free-energy of the conformational change of an amino acid from random to organized structures in lipid membranes (a) was used to cluster amino acids into 3 groups (b). These groups were used to encode peptide sequences as the relative position of the first amino acid in each group (c).

- Solvent accessibility (3 local features). Computed in the same way as the FET features, with amino acids groups representing levels of solvent accessibility.

- Amino acid composition (9 global features). As AMPs usually have biased amino compositions (Nagarajan et al., 2019; Jhong et al., 2019), we used the fraction of amino acids falling into 9 partially overlapping classes defined by charge, size, polarity, and hydrophobicity (see Methods and Suppl. Table S1).

- Charge (1 global feature). AMPs typically contain approximately 50\% hydrophobic residues (Zhang and Gallo, 2016; Malmsten, 2014; Pasupuleti et al., 2012), and their net charges are crucial to promote the peptide-induced membrane disruption (Malmsten, 2014; Pasupuleti et al., 2012; Ringstad et al., 2006).

- Membrane binding and solubility in different media (6 global features). These capture predisposition of peptides bind to membranes, and their solubility (Ebenhan et al., 2014; Dathe et al., 1997; Jhong et al., 2019).

All 22 descriptors used in Macrel are important for classification (see Suppl. Fig. S1). The fraction of acidic residues, charge, and isoelectric point were the most important variables in the hemolytic peptides classifier. Those variables tend to capture the electrostatic interaction between peptides and membranes, a key step in hemolysis. For AMP prediction, charge and the distribution parameters using FET and solvent accessibility are the most important variables. This is consistent with reports that cationic peptides (e.g., lysine-rich) show increased AMP activity (Bhadra et al., 2018; Jhong et al., 2019; Nagarajan et al., 2019). 


\subsection{Compared to other tools, Macrel achieves the highest specificity, albeit at lower sensitivity}

To evaluate the feature set and the classifier used in the context of the pipeline as a whole, we benchmark both the classifier implemented in Macrel, built with the training set adapted from Bhadra et al. (2018) (see Section 4.1.2), which consists of 1 AMP for each 50 negative examples, and a second AMP classifier (denoted Macrel $^{X}$ ), which was built using the same features and methods, but using the training set from Xiao et al. (2013), which contains 770 AMPs and 2405 non-AMPs (approximately $1: 3$ ratio).

Benchmark results show that the AMP classifier trained with a more balanced dataset performs better than most of alternatives considered, with AmPEP (Bhadra et al., 2018) achieving the best results (see Table 1).

In terms of overall accuracy, the AMP classifier implemented in Macrel is comparable to the best methods, with different trade-offs. In particular, Macrel achieves the highest precision and specificity at the cost of lower sensitivity. Although we do not possess good estimates of the proportion of AMPs in the smORFs predicted from real genomes (or metagenomes), we expect it to be much closer to 1:50 than to 1:3. Therefore, we chose to use the higher precision classifier in Macrel for AMP prediction from real data to minimize the number of false positives in the overall pipeline.

Table 1. The comparison of Macrel AMP classifier performance and state-of-art methods shows that Macrel is among the best methods across a range of metrics. The same test set (Xiao et al., 2013) was used to calculate the general performance statistics of the different classifiers, and the best value per column is in bold. Macrel refers to the Macrel classifier, while Macrel $^{X}$ is the same system trained with the Xiao et al. (2013) training set. Legend: Accuracy (Acc), Sensitivity (Sn), Specificity (Sp), Precision (Pr), and Matthew's Correlation Coefficient (MCC).

\begin{tabular}{lllllll}
\hline Method & Acc. & Sp. & Sn. & Pr. & MCC & Reference \\
\hline AmPEP $^{*}$ & $\mathbf{0 . 9 8}$ & - & - & - & $\mathbf{0 . 9 2}$ & Bhadra et al. (2018) \\
Macrel $^{X}$ & 0.95 & 0.97 & 0.94 & 0.97 & 0.91 & This study \\
iAMP-2L $_{\text {Macrel }}$ & 0.95 & 0.92 & 0.97 & 0.92 & 0.90 & Xiao et al. (2013) \\
AMAP & 0.95 & $\mathbf{0 . 9 9 8}$ & 0.90 & $\mathbf{0 . 9 9 8}$ & 0.90 & This study \\
CAMPR3-NN & 0.92 & 0.86 & 0.98 & 0.88 & 0.85 & Gull et al. (2019) \\
APSv2 & 0.80 & 0.71 & 0.89 & 0.75 & 0.61 & Waghu et al. (2016) \\
CAMPR3-DA & 0.78 & 0.57 & $\mathbf{0 . 9 9}$ & 0.70 & 0.61 & Veltri et al. (2018) \\
CAMPR3-SVM & 0.72 & 0.49 & 0.94 & 0.65 & 0.48 & Waghu et al. (2016) \\
CAMPR3-RF & 0.65 & 0.34 & 0.95 & 0.61 & 0.42 & Waghu et al. (2016) \\
iAMPpred & 0.64 & 0.32 & 0.96 & 0.59 & 0.39 & Waghu et al. (2016) \\
\hline
\end{tabular}

${ }^{*}$ These data were retrieved from the original paper.

AMPs, as they are likely to interact with cell membranes, can cause hemolysis, which can impact its potential uses, particularly in clinical settings (Zhang and Gallo, 2016; Ruiz et al., 2014; Oddo and Hansen, 2017). Therefore, for convenience, Macrel includes a classifier for hemolytic activity. This model is comparable to the state-of-the-art (see Table 2).

\subsection{High specificity is maintained when controlling for homology}

Although we used out-of-bag estimates (see Methods) to control for exact overlap between training and testing sets in the previous section, we still included similar sequences in training and testing, which would limit to an overestimate of performance. To control for this effect, Macrel and three methods (those where the ability to retrain the model was provided by the original authors) were tested using a stricter, homology-aware, scheme where training and testing datasets do not contain any homologous sequences between them ( $80 \%$ or higher identity, see Methods).

As expected, the measured performance was lower in this setting, but Macrel still achieved perfect specificity. Furthermore, this specificity was robust to changes in the exact proportion of AMPs:non-AMPs used in the training set, past a threshold (see Suppl. Table S2 and Fig. 3). Considering the overall performance of iAMP-2L model, future versions of Macrel could incorporate a combination of features from Macrel and iAMP-2L.

Using blastp as a classification method was no better than random, confirming that homology-based methods are not appropriate for this problem beyond very close homologues. 

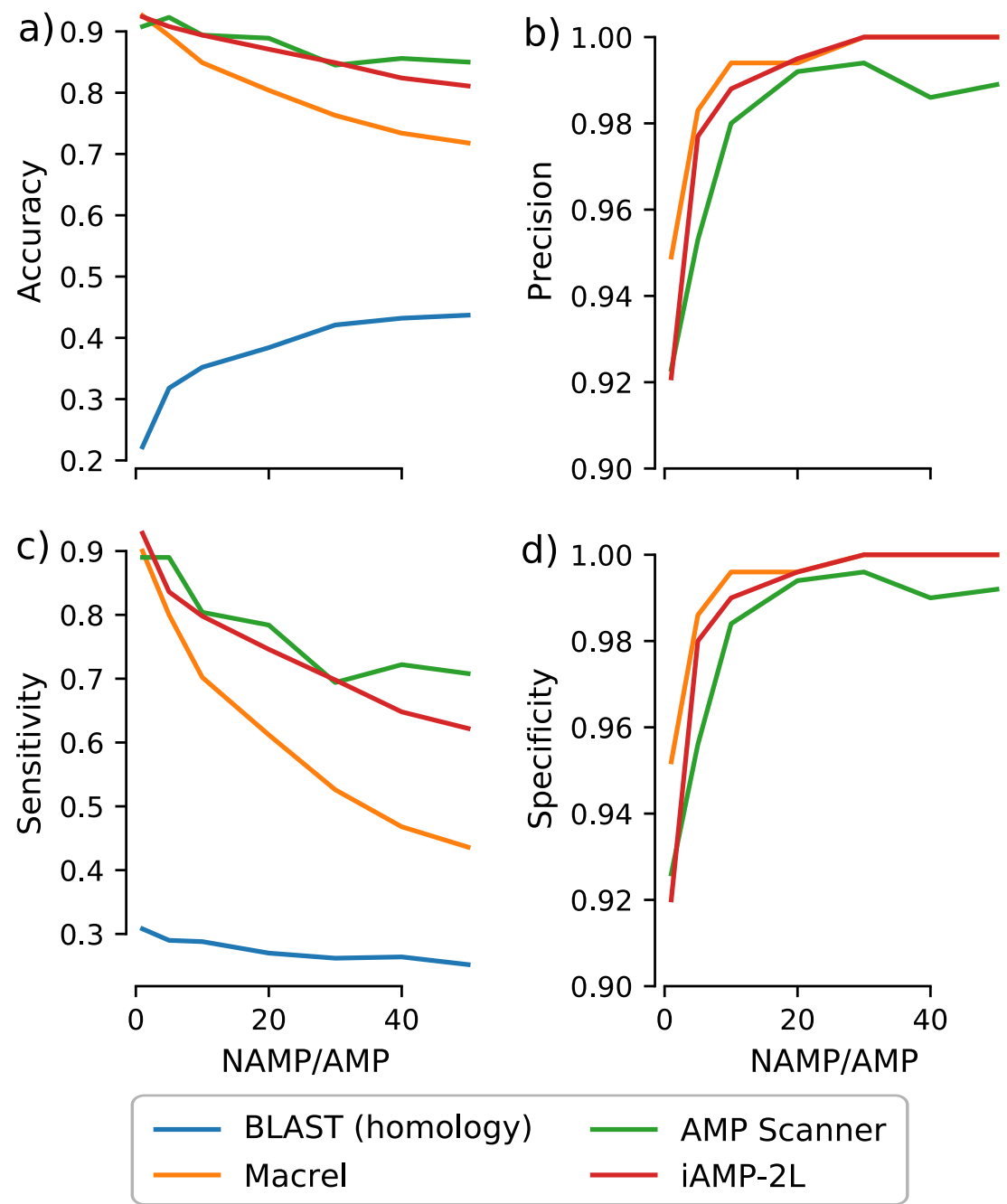

Figure 3. Specificity is maintained even when controlling for homology in training and testing. Different classifiers (blastp besthit as a purely homology-based system, Macrel, AMP Scanner v.2, and iAMP-2L) were trained with different proportions of non-AMPs:AMPs and tested on datasets which did not contain any homologs of sequences in the training set (using an 80\% identity cutoff). The results obtained in the homology-free datasets are showed in terms of accuracy (a), precision (b), Sensitivity (c), and Specificity (d), 
Table 2. Macrel achieves accuracy comparable to the state-of-art in hemolytic peptides classification. Models implemented by Chaudhary et al. (2016) were generically called HemoPI-1 due to the datasets used in the training and benchmarking (the best values per column are in bold).

\begin{tabular}{lllllll}
\hline Method & Acc. & Sp. & Sn. & Pr. & MCC & Reference \\
\hline HemoPI-1 $^{\text {C,SVM } *}$ & $\mathbf{0 . 9 5}$ & 0.95 & $\mathbf{0 . 9 6}$ & 0.95 & $\mathbf{0 . 9 1}$ & Chaudhary et al. (2016) \\
HemoPI-1 $^{\text {H } *}$ & $\mathbf{0 . 9 5}$ & 0.95 & $\mathbf{0 . 9 6}$ & 0.95 & $\mathbf{0 . 9 1}$ & Chaudhary et al. (2016) \\
HemoPI-1 $^{\text {C,IBK } *}$ & $\mathbf{0 . 9 5}$ & 0.94 & $\mathbf{0 . 9 6}$ & 0.94 & 0.89 & Chaudhary et al. (2016) \\
HemoPI-1 $^{\text {C,RF } *}$ & 0.94 & 0.95 & 0.94 & 0.95 & 0.89 & Chaudhary et al. (2016) \\
Macrel $^{\text {HemoPI-1 }}$ C,Log $*$ & 0.94 & $\mathbf{0 . 9 6}$ & 0.92 & $\mathbf{0 . 9 6}$ & 0.88 & This study \\
HemoPI-1 $^{\text {C,MP } *}$ & 0.94 & 0.94 & 0.93 & 0.94 & 0.87 & Chaudhary et al. (2016) \\
HemoPI-1 $^{\text {C,JK48 } *}$ & 0.89 & 0.93 & 0.94 & 0.93 & 0.87 & Chaudhary et al. (2016) \\
\hline
\end{tabular}

* These data were retrieved from the original paper.

\subsection{Macrel recovers a small number of high-quality AMP candidates per (meta)genome}

To evaluate Macrel on real data, we ran it on 484 reference genomes that had previously shown to be abundant in the human gut (Coelho et al., 2019). This resulted in 171,645 (redundant) smORFs. However, only 8,202 (after redundancy removal) of these were classified as potential AMPs. Spurio (Höps et al., 2018) classified 853 of these (circa 10\%) as likely spurious predictions.

Homology searches confirmed 13 AMP candidates as homologues from those in DRAMP database. Among them, a Laterosporulin (a bacteriocin from Brevibacillus), a BHT-B protein from Streptococcus, a Gonococcal growth inhibitor II from Staphylococcus, and other homologs of antimicrobial proteins. Seven of these confirmed AMPs were also present in the dataset used during model training.

To test Macrel on short-reads, we simulated metagenomes composed of these same 484 reference genomes, at three different sequencing depths (40,60, and 80 million reads) using abundance profiles estimated from 6 different real samples (Coelho et al., 2019) (for a total of 18 simulated metagenomes). The number of predicted smORFs increased with sequencing depth, with about 20k smORFs being predicted in the case of 80 million simulated reads (see Fig. 4). Despite this large number of smORF candidates, only a small portion of them $(0.17-0.64 \%)$ were classified as putative AMPs.

In total, we recovered 1,376 sequences for a total of 547 non-redundant AMPs predicted from the simulated metagenomes. Of these, only $44.5 \%$ are present in the underlying reference genomes. However, after eliminating singletons (sequences predicted in a single metagenome), this fraction rose to $80.4 \%$, which we thus recommend as a procedure to reduce false positives. Although fewer than half of pre-filtered AMP predictions were present in the reference genomes, only $12 \%$ of all predictions were marked as spurious by Spurio (see Methods). We manually investigated the origin of these spurious predictions and found that most of the spurious peptides are gene fragments from longer genes due to fragmentary assemblies or even artifacts of the simulated sequencing/assemblies. Interestingly, even the mispredictions were confirmed as AMPs by using the web servers of the methods tested in benchmark. In fact, circa $90 \%$ of all AMP candidates (including spurious predictions) were co-predicted by at least one other method than Macrel, and $61 \%$ were co-predicted by at least other 4 methods.

Having established that the rate of false positives can be kept low after singleton elimination, we investigated the recall of macrel, namely whether it was able to recover the AMPs that were present in the underlying genomes. Post hoc, we estimated that almost all (97\%) were in genomes with a coverage of at least 4.25 (while only 9\% of the non-recovered AMPs had this, or a higher, coverage, see Fig. 4). Nonetheless, in some exceptional cases, even very high coverage was not sufficient to recover a sequence.

\subsection{Macrel predicts putative AMPs in real human gut metagenomes}

To evaluate Macrel on real data, we used 182 previously published human gut metagenomes (Heintz-Buschart et al., 2016). Of these, 177 (97\%) contain putative AMPs, resulting in a total of 3,934 non-redundant sequences (see Suppl. Table S3). The fraction of smORFs classified as AMPs per metagenome ranged 0.1-1.65\%, a range similar to that observed in simulated metagenomes (see Section 2.5). 
a)

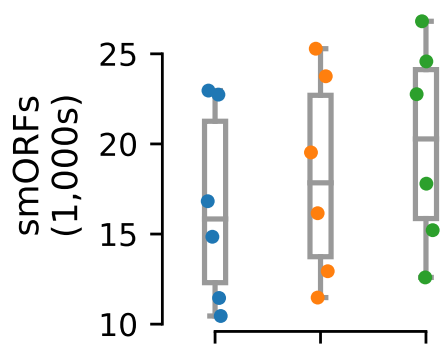

C)

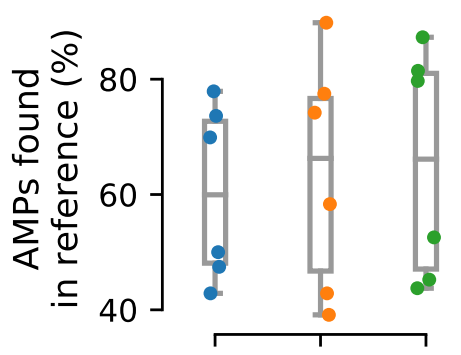

e)

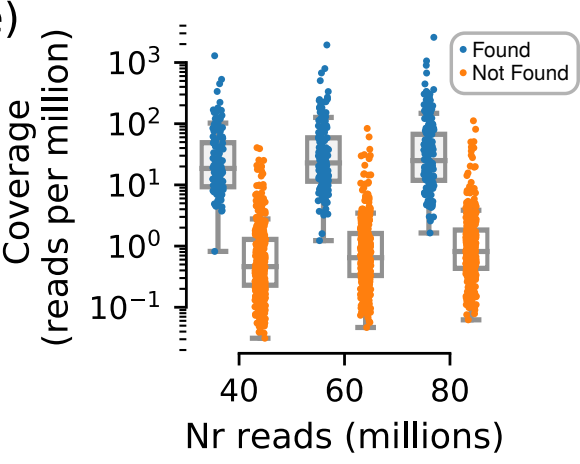

b)

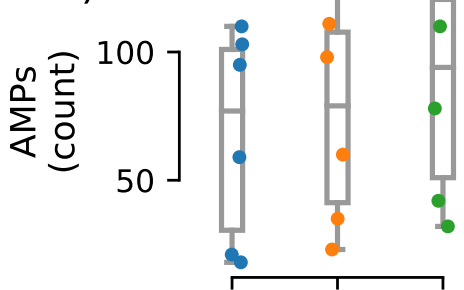

d)

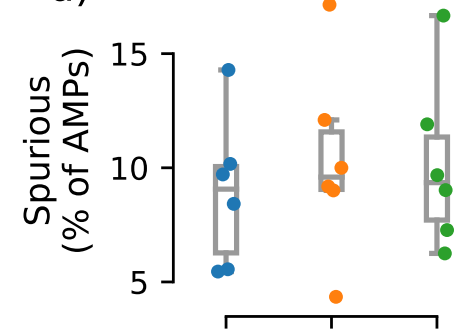

f)

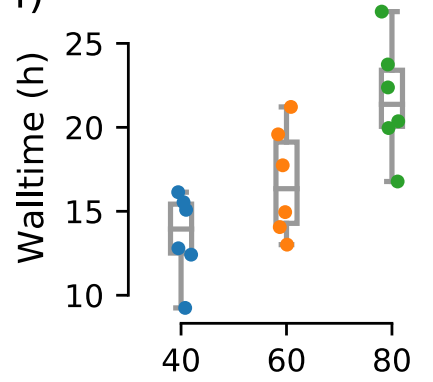

$\mathrm{Nr}$ reads (millions)

Figure 4. Macrel results in 6 different metagenome simulations involving variation of the number of reads (40-80 million). Six different microbial communities with realistic species abundances were simulated with increasing sequencing depth (see Methods). Macrel recovers a large number of small ORFs (smORFs) per metagenome (a), and a small number of AMPs from each metagenome (b). The number of AMPs returned that were present in the reference genomes covers a large range (40-90\%) (c), but only a small fraction is detected as being a spurious prediction (d) (see Methods). Detection of AMPs is heavily dependent on coverage (e), with almost all (97\%) of the detected AMPs contained in genomes with coverage above 4.25 (this is the simulated coverage of the genome, which, due to the stochastic nature of the process, will only correspond to the local coverage, on average). Processing times increase with coverage, with the single largest sample taking 27 hours (f). In all panels, boxplot whiskers represent 1.5 times the inter-quartile range (capped to the $0-100 \%$ range where appropriate) 
After eliminating singletons, 1,373 non-redundant AMP candidates remained, which we further tested with alternative methods. In total, $92.8 \%$ of the AMPs predicted with Macrel were also classified as such by at least one other classifier, and $65.5 \%$ of the times, half or more of the tested state-of-art methods agreed with Macrel results (see Suppl. Table S4). iAMPpred and CAMPR3-RF showed the highest agreement and co-predict 74.4\% and 65.7\% of the AMPs predicted by Macrel, respectively.

Ten percent of all predicted AMPs (414 peptides, or 10.5\%) were flagged as likely spurious (see Methods). The fraction of nonsingleton AMPs predicted as spurious was slightly lower (8\%, a non-significant difference). Our final dataset, after discarding both singletons and smORFs identified as spurious (see Methods and Suppl. Table S3), consists of 1,263 non-redundant AMPs.

As 36 metatranscriptomes produced from the same biological samples are also available, we quantified the expression of the 1,263 AMP candidates. Over 53.8\% of the predicted AMPs had detectable transcripts (see Suppl. Fig. S2). For 72\% of these, transcripts were detected in more than one metagenome.

Taken together, we concluded that Macrel could find a set of high-quality AMPs candidates, which extensively agrees with other state-of-art methods, many of which are being actively transcribed.

\subsection{Macrel requires only moderate computational resources}

The tests reported here were carried out on a personal laptop (32 GB of RAM and 4 cores) to show that Macrel is a pipeline with modest computational requirements. The execution time, although naturally dependent on the input size, was not greater than $27 \mathrm{~h}$ (recall that the largest simulated metagenomes contained 80 million reads). The reads trimming and assembly steps consumed $75-80 \%$ of the execution time, while gene prediction occupies another considerable part (10-15\%) (see Fig. 4).

\section{Conclusions}

Macrel performs all operations from raw metagenomic reads assembly to the prediction of AMPs. Using a combination of local and global sequence encoding techniques, Macrel classifiers perform comparably to the state-of-the-art in benchmark datasets. These benchmarks are valuable for method development, but as they contain the same number of AMP and non-AMP sequences in the testing set, are not a good proxy for the setting in which we intend to use the classifiers. It is unlikely that half of peptide sequences predicted from (meta)genomes will have antimicrobial activity. Therefore, we chose a classifier that achieves a slightly lower accuracy on these benchmarks, but has very high specificity.

The main challenge in computationally predicting smORFs (small ORFs, such as AMPs) with standard methods is the high rate of false-positives. However, after the filtering applied by Macrel classifiers, only a small number of candidate sequences remained. Supported by several lines of evidence (low level of detected spurious origin, similar classification by other methods, and evidence of AMPs transcription), we conclude that Macrel produces a set of high-quality AMP candidates.

Here, we presented an initial analysis of publicly-available human gut metagenomes (Heintz-Buschart et al., 2016). The 1,263 AMPs predicted with Macrel were largely congruent (92.8\%) with other state-of-art methods. This opens up the possibility of future work to understand the impact of these molecules on the microbial ecosystems or prospecting them for clinical or industrial applications.

Macrel is available as open-source software at https:/github.com/BigDataBiology/macrel and the functionality is also available as a webserver: http://big-data-biology.org/software/macrel.

\section{Methods}

\subsection{Macrel Classifiers}

\subsubsection{Features}

Local features, those dependent on the order of the peptide sequence, were inspired by the composition-transition-distribution (CTD) framework Dubchak et al. (1995, 1999). Physicochemical properties of a peptide at its $\mathrm{N}$ terminal are informative for the prediction of its antimicrobial activity (Bahar and Ren, 2013; Bhadra et al., 2018). Therefore, we defined features based on the normalized position of the first amino acid in a group of interest.

Global features, which are independent of amino acids primary sequence, were chosen to capture well-described AMP characteristics, such as the typical AMPs composition of approximately $50 \%$ hydrophobic residues, usual positive charge and folding into amphiphilic ordered structures (Zhang and Gallo, 2016). The mechanism of antimicrobial activity also was 
bioRxiv preprint doi: https://doi.org/10.1101/2019.12.17.880385; this version posted July 16, 2020. The copyright holder for this preprint (which was not certified by peer review) is the author/funder, who has granted bioRxiv a license to display the preprint in perpetuity. It is made available under aCC-BY-NC 4.0 International license.

summarized in Macrel's features by global descriptors of stability, amphiphilicity and predisposition of a peptide to bind to membranes.

Therefore, Macrel combines 6 local and 16 global features (see Suppl. Table S1), grouped as:

1. A new local feature group ( 3 local features), defined as the relative position of the first occurrence of residues in 3 groups of amino acids defined by their free energy of transition in a peptide from a random coil in aqueous environment to an organized helical structure in a lipid phase FET - see Fig. 2. The groups are: (1, lowest FET): ILVWAMGT, (2, intermediate): FYSQCN, (3, highest): PHKEDR (von Heijne and Blomberg, 1979).

2. Solvent Accessibility (3 local features), obtained by the distribution at first occurrence of residues organized in groups by solvent accessibility as described by Bhadra et al. (2018), using the groups: (1, buried): ALFCGIVW, (2, exposed): RKQEND, and (3, intermediate): MSPTHY.

3. Amino acid composition ( 9 global features) as the fraction of amino acids in groups defined by their size (area/volume), polarity, charge and R-groups: acidic, basic, polar, non-polar, aliphatic, aromatic, charged, small, tiny (Jhong et al., 2019; Nagarajan et al., 2019).

4. Charge and solubility (2 global features): peptide charge (Ebenhan et al., 2014; Chung et al., 2020) and isoelectric point (Fan et al., 2016; Wenzel et al., 2014; Chung et al., 2020).

5. Indexes for multiple purposes ( 3 global features): instability, aliphaticity, propensity to bind to membranes (Boman (Jhong et al., 2019; Chung et al., 2020; Boman, 2003)).

6. Hidrophobicity (2 global features): hydrophobicity (KyteDoolittle scale) and hydrophobic moment at $100^{\circ}$ to capture the helix momentum (Ebenhan et al., 2014; Dathe et al., 1997).

\subsubsection{Macrel prediction models}

For AMP prediction, our training set is adapted from the one presented by Bhadra et al. (2018) by eliminating redundant sequences. The resulting set contains 3,268 AMPs (from diverse databases, most bench-validated) and 165,138 non-AMPs (a ratio of approximately 1:50). A random forest classifier with 101 tree was trained using scikit-learn (Pedregosa et al., 2011) (all parameters, except the number of trees, were set to their default values).

The hemolytic activity classifier was built similarly to AMP classifier. For this, we used the training set HemoPI-1 from Chaudhary et al. (2016), which contains 442 hemolytic and 442 non-hemolytic peptides.

The datasets used in Macrel are extensively documented elsewhere (Bhadra et al., 2018; Xiao et al., 2013; Veltri et al., 2018; Chaudhary et al., 2016) and their description is available in the Suppl. Table S5. Briefly, the AMP dataset is formed by unique sequences collected from ADP3, CAMPR3, LAMP databases. Non-AMP sequences were retrieved from Uniprot database which were not annotated as AMP, membrane, toxic, secretory, defensing, antibiotic, anticancer, antiviral and antifungal. Hemolytic peptides dataset is composed of experimentally validated hemolytic peptides from Hemolytik database and randomly generated peptides from SwissProt as negative examples. No peptides containing non-canonical amino acids were kept.

\subsubsection{Prediction in (meta)genomes}

Macrel (see Fig. 1) accepts as inputs metagenomic paired-end or single-end reads in (possibly compressed) FastQ format and performs quality-based trimming with NGLess (Coelho et al., 2019). After this initial stage, Macrel assembles contigs using MEGAHIT ( $\mathrm{Li}$ et al., 2016) (a minimum contig length of 1,000 base pairs is used). Alternatively, if available, contigs can be passed directly to Macrel.

Genes are predicted on these contigs with a modified version of Prodigal (Hyatt et al., 2010), which predicts genes with a minimal length of 30 base pairs (compared to 90 base pairs in the standard Prodigal release). The original threshold was intended to minimize false positives (Hyatt et al., 2010), as gene prediction methods, in general, generate more false positives in shorter sequences (smORFs) (Höps et al., 2018). Sberro et al. (2019) showed that reducing the length threshold without further filtering could lead to as many as $61.2 \%$ of predicted smORFs being false positives. In Macrel, this filtering consists of outputting only those smORFs (10-100 amino acids) classified as AMPs.

For convenience, duplicated sequences can be clustered and output as a single entity. For calculating AMP abundance profiles, Macrel uses Paladin (Westbrook et al., 2017) and NGLess (Coelho et al., 2019). 
The gene prediction procedure inserts an initial methionine in the predicted peptides. However, there is not a proper system to predict when the $\mathrm{N}$-terminal methionine processing takes place in a given peptide. Thus, to avoid bias towards peptides containing or not initial methionine, Macrel was set to always exclude it prior features calculation.

\subsection{Benchmarking}

\subsubsection{Methods to be compared}

We compared the Macrel AMP classifier to the webserver versions of the following methods: CAMPR3 (including all algorithms) (Waghu et al., 2016), iAMP-2L (Xiao et al., 2013), AMAP (Gull et al., 2019), iAMPpred (Meher et al., 2017) and Antimicrobial Peptides Scanner v2 (Veltri et al., 2018). Results from AmPEP on this benchmark were obtained from the original publication (Bhadra et al., 2018). For all these comparisons, we used the benchmark dataset from Xiao et al. (2013), which contains 920 AMPs and 920 non-AMPs.

The datasets from (Xiao et al., 2013) do not overlap. However, the training set used in MACREL and the test set from Xiao et al. (2013) do overlap extensively. Therefore, for testing, after the elimination of identical sequences, we used the out-of-bag estimate for any sequences that were present in the training set. Furthermore, as described below, we also tested using an approach which avoids homologous sequences being present in both the testing and training (see Suppl. Table S5).

The benchmarking of the hemolytic peptides classifier was performed using the HemoPI-1 benchmark dataset formed by 110 hemolytic proteins and 110 non-hemolytic proteins previously established by Chaudhary et al. (2016). Macrel model performance was compared against models created using different algorithms (Chaudhary et al., 2016): Support vector machines-SVM, K-Nearest Neighbor (IBK), Neural networks (Multilayer Perceptron), Logistic regression, Decision trees (J48) and RF. There is no overlap between the training set and the testing set for the benchmark of hemolytic peptides.

\subsubsection{Homology-aware benchmarking}

Cd-hit (v4.8.1) (Fu et al., 2012) was used to cluster all sequences at $80 \%$ of identity and $90 \%$ of coverage of the shorter length. Only a single representative sequence from each cluster composed the dataset randomly split into training and testing partitions. The testing set was composed of 500 AMPs : 500 non-AMPs. The training set contained 1197 AMPs and was randomly selected to contain non-AMPs at different proportions (1:1, 1:5, 1:10, 1:20, 1:30, 1:40, 1:50).

Using the training and testing sets we tested 4 different methodologies: homology search, Macrel, iAMP-2L (Xiao et al., 2013) and AMP Scanner v.2 (Veltri et al., 2018) (these are the tools which enable users to retrain their classifiers). Homology search used blastp (Camacho et al., 2009), with a maximum e-value of 1e-5, minimum identity of 50\%, word size of 5, 90\% of query coverage, window size of 10 and subject besthit option. Sequences lacking homology were considered misclassified.

\subsubsection{Benchmarks on simulated and real data}

To test the Macrel short reads pipeline, 6 metagenomes were simulated at 3 different sequencing depths (40,60 and 80 million reads of $150 \mathrm{bp}$ ) with ART Illumina v2.5.8 (Huang et al., 2012) using the pre-built sequencing error profile for the HiSeq 2500 sequencer. To ensure realism, the simulated metagenomes contained species abundances estimated from real human gut microbial communities (Coelho et al., 2019).

We processed both the simulated metagenomes and the isolate genomes used to build the metagenomes with Macrel to verify whether the same AMP candidates could be retrieved and whether the metagenomic processing introduced false positive sequences not present in the original genomes.

The 182 metagenomes and 36 metatranscriptomes used for benchmarking were published by Heintz-Buschart et al. (2016) and are available from the European Nucleotide Archive (accession number PRJNA289586). Macrel was used to process metagenome reads (see Suppl. Table S3), and to generate the abundance profiles from the mapping of AMP candidates back to the metatranscriptomes. The results were transformed from counts to reads per million of transcripts.

\subsubsection{Detection of spurious sequences}

To test whether spurious smORFs still appeared in Macrel results, we used Spurio (Höps et al., 2018) and considered a prediction spurious if the score was greater or equal to 0.8 .

To identify putative gene fragments, the AMP sequences predicted with Macrel were validated through homology-searching against the non-redundant NCBI database (https://www.ncbi.nlm.nih.gov/). Predicted AMPs annotation was done by homology 
against the DRAMP database (Fan et al., 2016), which comprises circa 20k AMPs. The above-mentioned databases were searched with the blastp algorithm (Camacho et al., 2009), using a maximum e-value of $1 \cdot 10^{-5}$ and a word size of 3 . Hits with a minimum of $70 \%$ of identity and $95 \%$ query coverage were kept and parsed to the best-hits after ranking them by score, e-value, identity, and coverage. To check whether the AMPs predicted by the Macrel pipeline were gene fragments, patented peptides or known AMPs, the alignments were manually evaluated.

\subsection{Implementation and availability}

Macrel is implemented in Python 3, and R (R Core Team, 2018). Peptides (Osorio et al., 2015) is used for computing features, and the classification is performed with scikit-learn (Pedregosa et al., 2011). For ease of installation, we made available a bioconda package (Grüning et al., 2018). The source code for Macrel is archived at DOI:10.5281/zenodo.3608055 (with the specific version tested in this manuscript being available as DOI:10.5281/zenodo.3712125).

The complete set of scripts used to benchmark Macrel is available at https://github.com/BigDataBiology/macrel2020benchmark and the newly simulated generated dataset of different sequencing depths is available at Zenodo (DOI:10.5281/zenodo.3529860).

\section{Acknowledgments}

We thank Hiram He, Fudan University, who helped set up the Macrel website and kindly offered coding support as well as members of the Coelho group for helpful comments on previous versions of the manuscript. We thank beta users of macrel for their comments and bug reports.

\section{Funding}

This work was partly supported by National Natural Science Foundation of China (61932008, 61772368, 61572363), National Key R\&D Program of China (2018YFC0910500), Natural Science Foundation of Shanghai (17ZR1445600), Shanghai Municipal Science and Technology Major Project (2018SHZDZX01) and ZJLab.

\section{References}

A. Bahar and D. Ren. Antimicrobial peptides. Pharmaceuticals, 6(12):1543-1575, Nov 2013. ISSN 1424-8247. doi: 10.3390/ph6121543. URL http://dx.doi.org/10.3390/ph6121543.

G. Bernard, J. S. Pathmanathan, R. Lannes, P. Lopez, and E. Bapteste. Microbial dark matter investigations: How microbial studies transform biological knowledge and empirically sketch a logic of scientific discovery. Genome Biology and Evolution, 10(3):707-715, 2018. ISSN 1759-6653. doi: 10.1093/gbe/evy031. URL https://www.ncbi.nlm.nih.gov/pmc/ articles/PMC5830969/.

P. Bhadra, J. Yan, J. Li, S. Fong, and S. W. I. Siu. AmPEP: Sequence-based prediction of antimicrobial peptides using distribution patterns of amino acid properties and random forest. Scientific Reports, 8(1):1-10, 2018. ISSN 2045-2322. doi: 10.1038/s41598-018-19752-w.

H. G. Boman. Antibacterial peptides: basic facts and emerging concepts. Journal of Internal Medicine, 254(3):197-215, 2003.

K. Boone, K. Camarda, P. Spencer, and C. Tamerler. Antimicrobial peptide similarity and classification through rough set theory using physicochemical boundaries. BMC Bioinformatics, 19(1):469, 2018. ISSN 1471-2105. doi: 10.1186/ s12859-018-2514-6.

C. Camacho, G. Coulouris, V. Avagyan, N. Ma, J. Papadopoulos, K. Bealer, and T. L. Madden. BLAST+: architecture and applications. BMC bioinformatics, 10:421, 2009. ISSN 1471-2105. doi: 10.1186/1471-2105-10-421.

K. Chaudhary, R. Kumar, S. Singh, A. Tuknait, A. Gautam, D. Mathur, P. Anand, G. C. Varshney, and G. P. S. Raghava. A web server and mobile app for computing hemolytic potency of peptides. Scientific Reports, 6:22843, 2016. ISSN 2045-2322. doi: $10.1038 /$ srep22843.

C.-R. Chung, J.-H. Jhong, Z. Wang, S. Chen, Y. Wan, J.-T. Horng, and T.-Y. Lee. Characterization and identification of natural antimicrobial peptides on different organisms. International Journal of Molecular Sciences, 21(3):986, 2020. doi: 10.3390/ ijms21030986. URL https: / www. mdpi.com/1422-0067/21/3/986. Number: 3 Publisher: Multidisciplinary Digital Publishing Institute. 
L. P. Coelho, R. Alves, P. Monteiro, J. Huerta-Cepas, A. T. Freitas, and P. Bork. NG-meta-profiler: fast processing of metagenomes using NGLess, a domain-specific language. Microbiome, 7(1):84, 2019. ISSN 2049-2618. doi: 10.1186/ s40168-019-0684-8.

M. Dathe, T. Wieprecht, H. Nikolenko, L. Handel, W. L. Maloy, D. L. MacDonald, M. Beyermann, and M. Bienert. Hydrophobicity, hydrophobic moment and angle subtended by charged residues modulate antibacterial and haemolytic activity of amphipathic helical peptides. FEBS Letters, 403(2):208-212, 1997. ISSN 0014-5793. doi: 10.1016/S0014-5793(97)00055-0. URL http://www.sciencedirect.com/science/article/pii/s0014579397000550.

I. Dubchak, I. Muchnik, S. R. Holbrook, and S. H. Kim. Prediction of protein folding class using global description of amino acid sequence. Proceedings of the National Academy of Sciences of the United States of America, 92(19):8700-8704, 1995. ISSN 0027-8424.

I. Dubchak, I. Muchnik, C. Mayor, I. Dralyuk, and S. H. Kim. Recognition of a protein fold in the context of the structural classification of proteins (SCOP) classification. Proteins, 35(4):401-407, 1999. ISSN 0887-3585.

T. Ebenhan, O. Gheysens, H. G. Kruger, J. R. Zeevaart, and M. M. Sathekge. Antimicrobial peptides: Their role as infection-selective tracers for molecular imaging. BioMed Research International, 2014, 2014. ISSN 2314-6133. doi: 10.1155/2014/867381. URL https://www.ncbi.nlm.nih.gov/pmc/articles/PMC4163393/.

L. Fan, J. Sun, M. Zhou, J. Zhou, X. Lao, H. Zheng, and H. Xu. DRAMP: a comprehensive data repository of antimicrobial peptides. Scientific Reports, 6:24482, 2016. ISSN 2045-2322. doi: 10.1038/srep24482.

C. D. Fjell, H. Jenssen, K. Hilpert, W. A. Cheung, N. Panté, R. E. W. Hancock, and A. Cherkasov. Identification of novel antibacterial peptides by chemoinformatics and machine learning. Journal of Medicinal Chemistry, 52(7):2006-2015, 2009. ISSN 0022-2623. doi: 10.1021/jm8015365.

L. Fu, B. Niu, Z. Zhu, S. Wu, and W. Li. Cd-hit: accelerated for clustering the next generation sequencing data. Bioinformatics, 28:3150-3152, 2012. ISSN 1367-4803. doi: 10.1093/bioinformatics/bts565.

B. Grüning, R. Dale, A. Sjödin, B. A. Chapman, J. Rowe, C. H. Tomkins-Tinch, R. Valieris, J. Köster, and Bioconda Team. Bioconda: sustainable and comprehensive software distribution for the life sciences. Nature Methods, 15(7):475-476, 2018. ISSN 1548-7105. doi: 10.1038/s41592-018-0046-7.

S. Gull, N. Shamim, and F. Minhas. AMAP: Hierarchical multi-label prediction of biologically active and antimicrobial peptides. Computers in Biology and Medicine, 107:172-181, 2019. ISSN 1879-0534. doi: 10.1016/j.compbiomed.2019.02.018.

A. Heintz-Buschart, P. May, C. C. Laczny, L. A. Lebrun, C. Bellora, A. Krishna, L. Wampach, J. G. Schneider, A. Hogan, C. d. Beaufort, and P. Wilmes. Integrated multi-omics of the human gut microbiome in a case study of familial type 1 diabetes. Nature Microbiology, 2(1):1-13, 2016. ISSN 2058-5276. doi: 10.1038/nmicrobiol.2016.180.

W. Huang, L. Li, J. R. Myers, and G. T. Marth. ART: a next-generation sequencing read simulator. Bioinformatics (Oxford, England), 28(4):593-594, 2012. ISSN 1367-4811. doi: 10.1093/bioinformatics/btr708.

J. Huerta-Cepas, K. Forslund, L. P. Coelho, D. Szklarczyk, L. J. Jensen, C. von Mering, and P. Bork. Fast genome-wide functional annotation through orthology assignment by eggNOG-mapper. Molecular Biology and Evolution, 34(8):21152122, 2017. ISSN 1537-1719. doi: 10.1093/molbev/msx148.

D. Hyatt, G.-L. Chen, P. F. LoCascio, M. L. Land, F. W. Larimer, and L. J. Hauser. Prodigal: prokaryotic gene recognition and translation initiation site identification. BMC Bioinformatics, 11(1):119, 2010. ISSN 1471-2105. doi: 10.1186/ 1471-2105-11-119.

W. Höps, M. Jeffryes, and A. Bateman. Gene unprediction with spurio: A tool to identify spurious protein sequences. F1000Research, 7:261, 2018. ISSN 2046-1402. doi: 10.12688/f1000research.14050.1.

J.-H. Jhong, Y.-H. Chi, W.-C. Li, T.-H. Lin, K.-Y. Huang, and T.-Y. Lee. dbAMP: an integrated resource for exploring antimicrobial peptides with functional activities and physicochemical properties on transcriptome and proteome data. Nucleic Acids Research, 47:D285-D297, 2019. ISSN 0305-1048. doi: 10.1093/nar/gky1030.

S. Lata, N. K. Mishra, and G. P. S. Raghava. AntiBP2: improved version of antibacterial peptide prediction. BMC bioinformatics, 11 Suppl 1:S19, 2010. ISSN 1471-2105. doi: 10.1186/1471-2105-11-S1-S19. 
D. Li, R. Luo, C. M. Liu, C. M. Leung, H. F. Ting, K. Sadakane, H. Yamashita, and T. W. Lam. MEGAHIT v1.0: A fast and scalable metagenome assembler driven by advanced methodologies and community practices. Methods, 102:3-11, 2016. doi: 10.1016/j.ymeth.2016.02.020.

M. Malmsten. Antimicrobial peptides. Upsala Journal of Medical Sciences, 119(2):199-204, 2014. ISSN 0300-9734. doi: 10.3109/03009734.2014.899278.

P. K. Meher, T. K. Sahu, V. Saini, and A. R. Rao. Predicting antimicrobial peptides with improved accuracy by incorporating the compositional, physico-chemical and structural features into chou's general PseAAC. Scientific Reports, 7:42362, 2017. ISSN 2045-2322. doi: 10.1038/srep42362.

S. Miravet-Verde, T. Ferrar, G. Espadas-García, R. Mazzolini, A. Gharrab, E. Sabido, L. Serrano, and M. Lluch-Senar. Unraveling the hidden universe of small proteins in bacterial genomes. Molecular Systems Biology, 15(2):e8290, 2019. ISSN 1744-4292. doi: 10.15252/msb.20188290.

D. Nagarajan, T. Nagarajan, N. Nanajkar, and N. Chandra. A uniform in vitro efficacy dataset to guide antimicrobial peptide design. Data, 4(1):27, 2019. doi: 10.3390/data4010027.

A. Oddo and P. R. Hansen. Hemolytic activity of antimicrobial peptides. Methods in Molecular Biology (Clifton, N.J.), 1548: 427-435, 2017. ISSN 1940-6029. doi: 10.1007/978-1-4939-6737-7_31.

D. Osorio, P. Rondon-Villarreal, and R. Torres. Peptides: A package for data mining of antimicrobial peptides. The R Journal, 7(1):4-14, 2015.

F. Pascoal, C. Magalhães, and R. Costa. The link between the ecology of the prokaryotic rare biosphere and its biotechnological potential. Frontiers in Microbiology, 11, 2020. ISSN 1664-302X. doi: 10.3389/fmicb.2020.00231. URL https: //www.ncbi.nlm.nih.gov/pmc/articles/PMC7042395/.

M. Pasupuleti, A. Schmidtchen, and M. Malmsten. Antimicrobial peptides: key components of the innate immune system. Critical Reviews in Biotechnology, 32(2):143-171, 2012. ISSN 1549-7801. doi: 10.3109/07388551.2011.594423.

F. Pedregosa, G. Varoquaux, A. Gramfort, V. Michel, B. Thirion, O. Grisel, M. Blondel, P. Prettenhofer, R. Weiss, V. Dubourg, J. Vanderplas, A. Passos, D. Cournapeau, M. Brucher, M. Perrot, and E. Duchesnay. Scikit-learn: Machine learning in python. Journal of Machine Learning Research, 12:2825-2830, 2011.

R Core Team. R: The R project for statistical computing, 2018. URL https: / /www. r-project. org/. Accessed on 2019-12-17.

L. Ringstad, A. Schmidtchen, and M. Malmsten. Effect of peptide length on the interaction between consensus peptides and DOPC/DOPA bilayers. Langmuir: the ACS journal of surfaces and colloids, 22(11):5042-5050, 2006. ISSN 0743-7463. doi: $10.1021 / 1 a 060317 y$.

J. Ruiz, J. Calderon, P. Rondón-Villarreal, and R. Torres. Analysis of structure and hemolytic activity relationships of antimicrobial peptides (AMPs). In L. F. Castillo, M. Cristancho, G. Isaza, A. Pinzón, and J. M. C. Rodríguez, editors, Advances in Computational Biology, Advances in Intelligent Systems and Computing, pages 253-258. Springer International Publishing, 2014. ISBN 978-3-319-01568-2. doi: 10.1007/978-3-319-01568-2_36.

A. Saghatelian and J. P. Couso. Discovery and characterization of smORF-encoded bioactive polypeptides. Nature Chemical Biology, 11(12):909-916, 2015. ISSN 1552-4469. doi: 10.1038/nchembio.1964.

H. Sberro, B. J. Fremin, S. Zlitni, F. Edfors, N. Greenfield, M. P. Snyder, G. A. Pavlopoulos, N. C. Kyrpides, and A. S. Bhatt. Large-scale analyses of human microbiomes reveal thousands of small, novel genes. Cell, 178(5):1245-1259.e14, 2019. ISSN 0092-8674. doi: 10.1016/j.cell.2019.07.016.

A. Sharma, P. Gupta, R. Kumar, and A. Bhardwaj. dPABBs: A novel in silico approach for predicting and designing anti-biofilm peptides. Scientific Reports, 6:21839, 2016. ISSN 2045-2322. doi: 10.1038/srep21839.

N. Thakur, A. Qureshi, and M. Kumar. AVPpred: collection and prediction of highly effective antiviral peptides. Nucleic Acids Research, 40:W199-204, 2012. ISSN 1362-4962. doi: 10.1093/nar/gks450.

U. Theuretzbacher, K. Outterson, A. Engel, and A. Karlén. The global preclinical antibacterial pipeline. Nature Reviews Microbiology, pages 1-11, 2019. ISSN 1740-1534. doi: 10.1038/s41579-019-0288-0. 
D. Veltri, U. Kamath, and A. Shehu. Deep learning improves antimicrobial peptide recognition. Bioinformatics (Oxford, England), 34(16):2740-2747, 2018. ISSN 1367-4811. doi: 10.1093/bioinformatics/bty179.

G. von Heijne and C. Blomberg. Trans-membrane translocation of proteins. the direct transfer model. European Journal of Biochemistry, 97(1):175-181, 1979. ISSN 0014-2956. doi: 10.1111/j.1432-1033.1979.tb13100.x.

F. H. Waghu, R. S. Barai, P. Gurung, and S. Idicula-Thomas. CAMPR3: a database on sequences, structures and signatures of antimicrobial peptides. Nucleic Acids Research, 44:D1094-1097, 2016. ISSN 1362-4962. doi: 10.1093/nar/gkv1051.

M. Wenzel, A. I. Chiriac, A. Otto, D. Zweytick, C. May, C. Schumacher, R. Gust, H. B. Albada, M. Penkova, U. Krämer, R. Erdmann, N. Metzler-Nolte, S. K. Straus, E. Bremer, D. Becher, H. Brötz-Oesterhelt, H.-G. Sahl, and J. E. Bandow. Small cationic antimicrobial peptides delocalize peripheral membrane proteins. Proceedings of the National Academy of Sciences of the United States of America, 111(14):E1409-1418, 2014. ISSN 1091-6490. doi: 10.1073/pnas.1319900111.

A. Westbrook, J. Ramsdell, T. Schuelke, L. Normington, R. D. Bergeron, W. K. Thomas, and M. D. MacManes. PALADIN: protein alignment for functional profiling whole metagenome shotgun data. Bioinformatics (Oxford, England), 33(10): 1473-1478, 2017. ISSN 1367-4811. doi: 10.1093/bioinformatics/btx021.

X. Xiao, P. Wang, W.-Z. Lin, J.-H. Jia, and K.-C. Chou. iAMP-2l: a two-level multi-label classifier for identifying antimicrobial peptides and their functional types. Analytical Biochemistry, 436(2):168-177, 2013. ISSN 1096-0309. doi: 10.1016/j.ab. 2013.01.019.

L.-J. Zhang and R. L. Gallo. Antimicrobial peptides. Current biology: CB, 26(1):R14-19, 2016. ISSN 1879-0445. doi: 10.1016/j.cub.2015.11.017. 